\title{
Verification of a simplified mathematical model of centrifugal compressor stages
}

\author{
A. Rekstin ${ }^{1, *}, K$. Soldatova ${ }^{1}, Y$. Galerkin ${ }^{1}$, and E. Popova ${ }^{1}$ \\ ${ }^{1}$ Peter the Great St. Petersburg Polytechnic University, St. Petersburg, Russia
}

\begin{abstract}
To calculate the efficiency of a centrifugal compressor, it is sufficient to know the design parameters and similarity criteria: flow rate coefficient, loading factor, relative hub ratio, Mach number. The effect of the inlet nozzle and the diffuser type is also taken into account. The original simplified model was successfully used for calculation of compressors' candidates in computer programs of the Universal Modelling Method. Recently, the model has undergone significant revision and been remade. The modernized model is used in the program for primary design of centrifugal compressors. The authors verified the new model, comparing the calculated efficiency with the measured efficiency of several dozens of model stages $21 \mathrm{CV}$ family and low flow rate model stages. In total, calculations were carried out for more than thirty model stages. The range of design parameters of analysed model stages is quite wide: flow rate coefficient $0.00564-0.0676$; loading factor $0.384-0.742$; hub ratio $0.258-0.466$.
\end{abstract}

\section{Introduction}

A designer of an industrial compressor can choose between the number of rotors and their RPM, the number of stages on each rotor, diameters of impellers, types of impellers (2D or 3D), types of diffusers (vane (VD), vaneless (VLD)), number and position of intermediate coolers.

Modern methods of gas-dynamic design are based on mathematical models (MM) of efficiency and head. The authors apply the Universal Modelling Method in design practice, the foundations of which are presented in the monograph [1]. The Method is able to design a flow part and calculate the gas dynamic characteristics of any of the possible candidates of the compressor listed above. However, this way of candidates' choice would be too complicated. For better selection of a candidate, an easier method is needed.

\section{Purpose of the simplified mathematical model of centrifugal compressor stage efficiency}

Equations listed below demonstrate that in order to calculate compressor candidate's efficiency and impeller diameters, only the stages' efficiency is required. The purpose of the simplified model is to predict stage efficiency, if a stage design flow rate coefficient, design loading factor, hub ratio and Mach number are known:

- flow rate coefficient at design mass flow rate

$$
\Phi_{\text {des }}=\frac{\bar{m}_{\text {des }}}{\frac{\mathrm{p}_{0}^{*}}{\mathrm{PT}_{0}^{*}} \frac{\pi}{4} D_{2}^{2} u_{2}}
$$

- loading factor at design mass flow rate

$$
\psi_{\mathrm{Tdes}}=c_{u 2 \mathrm{des}} / u_{2}
$$

- hub ratio

$$
\overline{\mathrm{D}}_{\mathrm{h}}=\frac{\mathrm{D}_{\mathrm{h}}}{\mathrm{D}_{2}}
$$

- Mach number

$$
M_{u}=u_{2} / \sqrt{k R T_{0}^{*}}
$$

where $\bar{m}$ is mass flow rate, $p^{*}$ is total pressure, $R$ is gas constant,$T^{*}$ is total temperature, $D_{2}$ is impeller diameter, $c_{u 2 \text { des }}$ is circumferential component of absolute velocity at impeller exit, $u_{2}$ is blade velocity , des-.designed, 0 - impeller entrance.

\section{Equations for main parameters of compressor candidates}

Equation (5) defines flow rate coefficients of stages with mass flow rate of the compressor:

\footnotetext{
Corresponding author: rekstin2k7@ mail.ru
} 


$$
\begin{aligned}
& \bar{m}_{\mathrm{des}}=\bar{m}_{\mathrm{des} 1}=\ldots=\bar{m}_{\mathrm{des} z}=\Phi_{\mathrm{des} 1}\left(\frac{p_{01}^{*}}{R T_{01}^{*}} D_{2}^{2} u_{2}\right)_{\operatorname{des} 1}= \\
& =\ldots=\Phi_{\operatorname{des} z}\left(\frac{p_{0 \mathrm{z}}^{*}}{R T_{0 \mathrm{z}}^{*}} D_{2}^{2} u_{2}\right)_{\operatorname{des} z}
\end{aligned}
$$

Blade velocity:

$$
\mathrm{u}_{2}=\frac{\pi \mathrm{D}_{2} \mathrm{n}}{60}(\mathrm{~m} / \mathrm{s})
$$

where $\mathrm{n}$ is a number of rotor rotation per minute.

Flow rate coefficients of stages located on the same rotor:

$$
\begin{aligned}
& m_{\text {des }} /\left(0,05235 \rho_{0(1)}^{*} D_{2}^{3} n\right)_{\text {des } 1}=\Phi_{\text {des } 1}= \\
& \Phi_{\operatorname{des} 2} \frac{\rho_{0 \operatorname{des}(2)}^{*}}{\rho_{0(1)}^{*}}\left(D_{2(2)} / D_{2(1)}\right)^{3}=\Phi_{\operatorname{des} 1} \frac{\rho_{0 \operatorname{des}(\mathrm{i})}^{*}}{\rho_{0(1)}^{*}}\left(D_{2(\mathrm{i})} / D_{2(1)}\right)^{3}
\end{aligned}
$$

In equation (7) the ratio of the diameters of the first stage on the shaft is the investigated parameter of the candidates'.

Compressibility factor for the first stage:

$\varepsilon_{1}=\frac{\rho_{0 \operatorname{des}(2)}^{*}}{\rho_{0(1)}^{*}}=\left(\frac{\rho_{0}^{*}}{R T_{0}^{*}}\right)_{2} /\left(\frac{\rho_{0}^{*}}{R T_{0}^{*}}\right)_{1}=\left(\frac{T_{0(2)}^{*}}{T_{0(1)}^{*}}\right)^{\left(\frac{1}{n-1}\right)_{1}}$

The stage polytrophic index depends on isentropic coefficient and efficiency: $n \sqcup \frac{\eta^{*} \frac{k}{k-1}}{\eta^{*} \frac{k}{k-1}-1}$ where $\eta^{*}$ is polytrophic efficiency by total parameters: $\eta^{*}=\frac{\ln \frac{\mathrm{p}_{0^{\prime}}^{*}}{\mathrm{p}_{0}^{*}}}{\frac{\mathrm{k}}{\mathrm{k}-1} \ln \frac{\mathrm{T}_{0^{\prime}}^{*}}{\mathrm{~T}_{0}^{*}}}$ where $0^{\prime}$ is the exit of the stage.

The ratio of total temperatures from the energy equation:

$$
\left(\frac{T_{0(2)}^{*}}{T_{0(1)}^{*}}\right)_{\text {des }}=\frac{T_{0(1)}^{*}+\frac{\psi_{i(1) \operatorname{des}} u_{2(1)}^{2}}{\frac{k}{k-1} R}}{T_{0(1)}^{*}}
$$

The work coefficient $\psi_{i}$ depends on the loading factor $\psi_{T}$ and coefficients of disc friction $\beta_{d f}$ and leakage coefficient $\beta_{l k}$ in labyrinth seals.

$$
\psi_{i}=\psi_{\mathrm{T}}\left(1+\beta_{\mathrm{df}}+\beta_{\mathrm{lk}}\right)
$$

The loading factor $\psi_{\text {Tdes }}$ is the design parameter chosen by the designer. The semi-empirical equations checked by the practice of calculations are used in the Universal Modeling Method [2]:

$$
\beta_{d f}=K_{d f} \frac{\varepsilon_{2}}{22.68 \cdot \Phi \psi_{\mathrm{T}}} \beta_{l k}=K_{l k} \frac{\bar{\delta}_{\text {seal }}}{\Phi} \frac{\bar{D}_{1}}{K_{D}}
$$

where $K_{d f}, K_{l k}$ are the empirical coefficients, $\bar{\delta}_{\text {seal }}$ is the gap in the labyrinth seal, referred to $D_{2}$.

The ratio of stage pressures from the process equation:

$$
\pi^{*}=\left(\frac{T_{2}^{*}}{T_{0}^{*}}\right)^{\frac{k}{k-1} \eta^{*}}=\left(1+\frac{h_{i}}{\frac{k}{k-1} R T_{0}^{*}}\right)^{\frac{k}{k-1} \eta^{*}}
$$

The internal head of the compressor:

$$
H_{\text {ides }}=\frac{k}{k-1} R T_{\text {н }}^{*}\left(\pi_{\text {des }}^{*-1}-1\right)^{\frac{k}{k-1} \eta^{*}}=\sum_{1}^{z} \psi_{i} u_{2}^{2}
$$

where: $\pi_{\mathrm{des}}=\left(\pi_{1}^{*} \times \pi_{2}^{*} \times \ldots \times \pi_{z}^{*}\right)_{\text {des }}$.

Compressor shaft power:

$$
N_{i}=H_{i} \times \bar{m}
$$

To calculate compressor candidates with a given mass flow rate $\overline{\mathrm{m}}_{\mathrm{des}}$ and pressure ratio $\pi_{\mathrm{des}}^{*}$, one must know the temperature and pressure at the compressor inlet $T_{i n l}^{*}=T_{01}^{*}, \mathrm{p}_{\text {inl }}^{*}$ the isentropic index $k=c_{p} / c_{v}$ and the gas constant $\mathrm{R}(\mathrm{J} / \mathrm{kg} / \mathrm{K})$. Taking into account the pressure losses in the inlet nozzle, the total pressure $p_{0}^{*}$ at the inlet to the 1 st stage should be calculated. For candidates with internal coolers pressure loss is taken into account.

Iterative process for equations (1-15) defines values of $\Phi_{\text {des }}, \psi_{\text {Tdes }}, \overline{\mathrm{D}}_{\mathrm{h}}, \mathrm{M}_{\mathrm{u}}$ that are sufficient to calculate $\Phi_{\text {des }}$ by the simplified math model. The results of numerical simulation were compared with experimental data.

\section{A simplified mathematical model of a centrifugal stage efficiency}

The original variant of the simplified math model is presented in paper [3]. Still in [4] was presented the procedure where dimensions and configuration of a stage are connected with design parameters $\Phi_{\text {des }}, \psi_{\text {Tdes }}$, $\overline{\mathrm{D}}_{\mathrm{h}}, \mathrm{M}_{\mathrm{u}}, \mathrm{k}$. But the dimensions and similarity criteria 
determine stage's efficiency. Loss of efficiency $\eta_{\text {imp }}^{*}$ in an impeller and in stator $\Delta \eta_{\mathrm{st}}^{*}$ of a stage:

$$
\begin{gathered}
\Delta \eta_{\mathrm{imp}}^{*}=\frac{\zeta_{\mathrm{imp}}}{2 \psi_{\mathrm{T}}\left(1+\beta_{\mathrm{df}}+\beta_{\mathrm{lk}}\right)}\left(\frac{\mathrm{w}_{1}}{\mathrm{u}_{2}}\right)^{2}+\frac{\beta_{\mathrm{df}}+\beta_{\mathrm{lk}}}{1+\beta_{\mathrm{df}}+\beta_{\mathrm{lk}}} \\
\Delta \eta_{\mathrm{st}}^{*}=\frac{\zeta_{\mathrm{st}}}{2 \psi_{\mathrm{T}}\left(1+\beta_{\mathrm{df}}+\beta_{\mathrm{lk}}\right)}\left(\frac{\mathrm{c}_{2}}{\mathrm{u}_{2}}\right)^{2}
\end{gathered}
$$

where: $\zeta_{\text {imp }}, \zeta_{\text {st }}$ are loss coefficients of impeller and stator part of a stage, $\mathrm{W}_{1}$ is relative velocity at an impeller blade cascade inlet, $c_{2}$ is absolute velocity at a stator part inlet.

The simplified math model exploit the fact that when $\Phi_{\text {des }}<0.034-0.040$ flow part channels become narrower, and surface friction diminish efficiency. The second factor - parasitic loss coefficient $\beta_{\mathrm{df}}+\beta_{\mathrm{lk}}$ becomes bigger.

The bigger $\Phi_{\text {des }}$ is the bigger is an impeller inlet diameter. It leads to bigger $\mathrm{w}_{1}$. When $\Phi_{\text {des }}>0.09-$ 0.11 , the efficiency decline is inevitable.

The bigger $\overline{\mathrm{D}}_{\mathrm{h}}$ is the bigger is an impeller inlet diameter. It also leads to bigger $\mathrm{W}_{1}$ and a decline in efficiency.

The bigger $\psi_{\mathrm{Tdes}}$ is the bigger is velocity at a stator part inlet. The second factor is that bigger $\psi_{\text {Tdes }}$ demands higher blade load and more intensive deceleration. Loss of efficiency takes place in both elements of a stage.

With the growth of Mach number there is a tendency of efficiency decrease due to averaged and local flow deceleration. The simplified model takes it into account too.

The description of the new simplified model is presented in [5]. The general equation of the model is:

$$
\eta_{\text {des }}^{*}=1-X_{1} K_{\Phi} K_{\psi_{\mathrm{T}}} K_{D_{h u b}} K_{\mathrm{M}}+\Delta \eta_{v d}-\Delta \eta_{i p},
$$

where $X_{1}$ and other $X$ below are empirical coefficients of the math model. Equation (21) is a system of equations for correction coefficients $K$ that reflects influence of $\Phi_{\text {des }}, \psi_{\text {Tdes }}, \overline{\mathrm{D}}_{\mathrm{h}}, \mathrm{M}_{\mathrm{u}}$.

Correction coefficient $\mathrm{K}_{\Phi}$ : at $\Phi_{\text {des }} \leq 0.085$ : $\mathrm{K}_{\Phi}=1+\mathrm{X}_{2}\left[\mathrm{X}_{3}\left(0.085-\Phi_{\mathrm{des}}\right)\right]^{\mathrm{X}_{4}}$;

At

$$
\Phi_{\mathrm{des}}>0.085
$$

$\mathrm{K}_{\Phi}=1+\mathrm{X}_{5}\left(\Phi_{\mathrm{des}}-0.085\right)^{\mathrm{X}_{6}}\left(1+\mathrm{X}_{7} \overline{\mathrm{D}}_{\text {hub }}^{\mathrm{X}_{8}}\right)$.

Correction coefficient
$\mathrm{K}_{\psi_{\mathrm{T}}}=1+\mathrm{X}_{9}\left(\psi_{\mathrm{Tdes}}-0.5\right)^{\mathrm{X}_{10}}$ Correction coefficient $\mathrm{K}_{\mathrm{D}_{\text {hub }}}: \mathrm{K}_{\mathrm{D}_{\text {hub }}}=1+\mathrm{X}_{11} \overline{\mathrm{D}}_{\text {hub }}^{\mathrm{X}_{12}}\left(1+\Phi_{\text {des }}\right)^{\mathrm{X}_{13}}$;

The correction coefficient $K_{\mathrm{M}}$ : $\mathrm{K}_{\mathrm{M}_{\mathrm{u}}}=1+\mathrm{X}_{14}\left(\mathrm{M}_{\mathrm{u}}-0.5\right)^{\mathrm{X}_{15}}(\Phi-0.01)^{\mathrm{X}_{16}}$. The loss of efficiency in an inlet nozzle: $\Delta \eta_{\mathrm{ip}}=\mathrm{X}_{17} \frac{\Phi_{\mathrm{des}}^{\mathrm{X}_{18}}}{\psi_{\mathrm{Tdes}}}$. Efficiency gain if a vane diffusor is installed instead of vaneless diffuser: $\Delta \eta_{\mathrm{vd}}=\mathrm{X}_{19}\left(\psi_{\text {Tdes }}-0.50\right)^{\mathrm{X}_{20}}$. To apply the system of equations (21) in design practice, it is necessary to verify the simplified math model.

\section{Objects and results of verification}

The results of simulation of the gas-dynamic characteristics of 20 pipe line compressors 4-25 MW (delivery pressure of up to $12 \mathrm{MPa}$ ) are presented in [6]. The compressors have 2 to 6 stages, vaneless and vane diffusers. Figure 1 demonstrates that a compressor flow part consists of three types of stages:

- suction stage "impeller+diffuser+return channel”,

- suction stage "impeller+diffuser+exit nozzle".

The characteristics' simulation was made by the most modern 8th version of the Universal Modeling Method [2, 7-12].

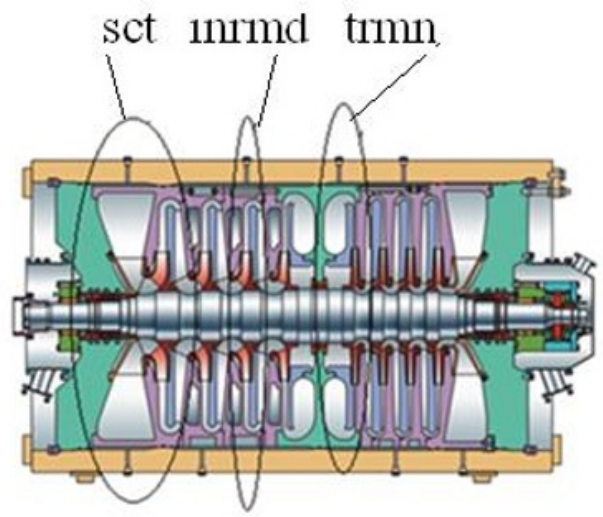

Fig. 1. Suction, intermediate and terminal stages of a multistage compressor.

In Table 1 we compare the maximum efficiency obtained through factory testing and by simulating 20 pipe line compressors with different parameters [13]. The compressors were produced by different Russian and foreign manufacturers. The factory test data were presented by the manufacturers to the author of [13] for the Universal Modeling Method promotion.

The properties of the compressors based on the \#1: volumetric flow rate $573 \mathrm{~m}^{3} / \mathrm{min}$, delivery pressure 76 at (abs), pressure ratio 1.54. Compressors \#\#1-18 were designed using the Universal modeling Method. The origin of compressors \#\#19, 20 designs is unknown. The accuracy of simulation compressors \#\#1 - 18 is quite satisfactory. 
Table 1. Measured and simulated efficiency of 20 pipe line compressors.

\begin{tabular}{|l|l|l|l|l|l|}
\hline № & Name & diffusor & $\eta_{\max .}(\exp )$ & $\eta_{\max }(\operatorname{sim})$ & $\eta_{\max \exp }-\eta_{\max \text { des }}$ \\
\hline 1 & $248-76-1.72$ & VD & 0.800 & 0.799 & +0.001 \\
\hline 2 & $573-76-1.54$ & VLD & 0.845 & 0.848 & -0.003 \\
\hline 3 & $350-73-1.39$ & VLD & 0.854 & 0.841 & +0.013 \\
\hline 4 & $338-76-1.45$ & VLD & 0.839 & 0.844 & -0.005 \\
\hline 5 & $249-76-1.71$ & VLD & 0.875 & 0.868 & +0.007 \\
\hline 6 & $385-61-1.67$ & VLD & 0.805 & 0.791 & +0.014 \\
\hline 7 & $369-76-1.48$ & VLD & 0.855 & 0.844 & +0.011 \\
\hline 8 & $338-76-1.5$ & VLD & 0.837 & 0.846 & -0.009 \\
\hline 9 & $56-76-1.77$ & VLD & 0.843 & 0.836 & +0.007 \\
\hline 10 & $53-125-1.95$ & VLD & 0.789 & 0.805 & -0.016 \\
\hline 11 & $450-41-2.25$ & VD & 0.801 & 0.814 & -0.013 \\
\hline 12 & $470-45-1.76$ & VLD & 0.827 & 0.828 & -0.001 \\
\hline 13 & $151-64-1.7$ & VLD & 0.813 & 0.821 & -0.008 \\
\hline 14 & $146-71-1.75$ & VLD & 0.806 & 0.818 & -0.012 \\
\hline 15 & $106-82-1.85$ & VLD & 0.811 & 0.820 & -0.009 \\
\hline 16 & $94-77-1.7$ & VLD & 0.811 & 0.823 & -0.012 \\
\hline 17 & $121-107-1.55$ & VLD & 0.794 & 0.809 & -0.015 \\
\hline 18 & $151-64-1.7$ & VLD & 0.813 & 0.821 & -0.008 \\
\hline 19 & $31-100-2.28$ & VLD & 0.77 & 0.795 & -0.025 \\
\hline 20 & $175-56-1.61$ & VD & 0.818 & 0.856 & -0.038 \\
\hline
\end{tabular}

The author of the [13] have calculated characteristics of compressors' stages using the same $8^{\text {th }}$ version of the Universal Modeling Method mathematical model. There is the information on 70 suction, intermediate and terminal stages in the database of model stages of the family $21 \mathrm{CV}$. The family $21 \mathrm{CV}$ stages range of design parameters: $\Phi_{\text {des }}=\Phi_{\text {opt }}=0.0202-0.0676, \Psi_{\text {des }}=0.384-$
$0.742, \overline{\mathrm{D}}_{\mathrm{h}}=0.258-0.466$.

Parameters of intermediate model stages of the family $21 \mathrm{CV}$ and maximum efficiency calculated by simplified math model are presented in the Table 2 . Verification results for suction and terminal; stages are of the same character.

Names of the family $21 \mathrm{CV}$ stages are in column 2 .

Table 2. Parameters of intermediate model stages of the family $21 \mathrm{CV}$ and maximum efficiency calculated by simplified math model.

\begin{tabular}{|c|c|c|c|c|c|c|c|}
\hline № & Stage & $\Phi_{\text {des }}$ & $\Psi_{\text {Tdes }}$ & $\bar{D}_{\mathrm{h}}$ & $\eta_{\max }^{*} 21 \mathrm{CV}$ & $\eta_{\text {max }}^{*}$ s.m. & $\eta_{2 I C V}^{*} / \eta_{\text {sm }}^{*}$ \\
\hline $\mathbf{1}$ & med 023/383 & 0.0226 & 0.3832 & 0.409 & 0.807 & 0.759 & 1.063 \\
\hline $\mathbf{2}$ & med 023/384 & 0.0232 & 0.3836 & 0.466 & 0.787 & 0.755 & 1.042 \\
\hline $\mathbf{3}$ & med 026/371 & 0.0256 & 0.3708 & 0.483 & 0.791 & 0.763 & 1.037 \\
\hline $\mathbf{4}$ & med 028/379 & 0.0279 & 0.3796 & 0.378 & 0.826 & 0.784 & 1.054 \\
\hline $\mathbf{5}$ & med 035/561 & 0.0348 & 0.5608 & 0.35 & 0.807 & 0.808 & 0.999 \\
\hline $\mathbf{6}$ & med 037/551 & 0.037 & 0.5512 & 0.35 & 0.815 & 0.815 & 1.0 \\
\hline $\mathbf{7}$ & med 041/571 & 0.0414 & 0.571 & 0.3405 & 0.849 & 0.827 & 1.027 \\
\hline $\mathbf{8}$ & med 042/463 & 0.0416 & 0.4628 & 0.371 & 0.868 & 0.826 & 1.051 \\
\hline $\mathbf{9}$ & med 042/520 & 0.042 & 0.5204 & 0.344 & 0.85 & 0.829 & 1.025 \\
\hline $\mathbf{1 0}$ & med 042/561 & 0.0424 & 0.5607 & 0.3405 & 0.844 & 0.829 & 1.018 \\
\hline $\mathbf{1 1}$ & med 043/546 & 0.0426 & 0.546 & 0.339 & 0.856 & 0.831 & 1.041 \\
\hline $\mathbf{1 2}$ & med 043/467 & 0.043 & 0.4674 & 0.371 & 0.869 & 0.83 & 1.047 \\
\hline $\mathbf{1 3}$ & med 044/555 & 0.0443 & 0.5554 & 0.35 & 0.821 & 0.833 & 0.986 \\
\hline $\mathbf{1 4}$ & med 045/470 & 0.0446 & 0.4696 & 0.371 & 0.872 & 0.833 & 1.047 \\
\hline $\mathbf{1 5}$ & med 045/699 & 0.0455 & 0.6991 & 0.37 & 0.801 & 0.827 & 0.967 \\
\hline $\mathbf{1 6}$ & med 046/565 & 0.0458 & 0.5645 & 0.344 & 0.859 & 0.837 & 1.026 \\
\hline $\mathbf{1 7}$ & med 056/598 & 0.0561 & 0.5977 & 0.34 & 0.854 & 0.853 & 1.001 \\
\hline $\mathbf{1 8}$ & med 056/451 & 0.0563 & 0.4506 & 0.323 & 0.886 & 0.857 & 1.034 \\
\hline $\mathbf{1 9}$ & med 059/52 & 0.059 & 0.5196 & 0.339 & 0.876 & 0.859 & 1.020 \\
\hline $\mathbf{2 0}$ & med 061/635 & 0.061 & 0.6345 & 0.314 & 0.874 & 0.859 & 1.017 \\
\hline $\mathbf{2 1}$ & med 061/482 & 0.0612 & 0.4824 & 0.287 & 0.891 & 0.865 & 1.030 \\
\hline $\mathbf{2 2}$ & med 063/418 & 0.0627 & 0.4184 & 0.337 & 0.876 & 0.863 & 1.015 \\
\hline $\mathbf{2 3}$ & med 063/441 & 0.0633 & 0.4411 & 0.323 & 0.888 & 0.8645 & 1.027 \\
\hline $\mathbf{2 4}$ & med 065/646 & 0.0651 & 0.646 & 0.322 & 0.838 & 0.862 & 0.972 \\
\hline $\mathbf{2 5}$ & med 066/458 & 0.0664 & 0.4583 & 0.258 & 0.879 & 0.871 & 1.009 \\
\hline & & & & & & & \\
\hline
\end{tabular}


Name of the stage \#1 means: $\Phi_{\text {des }}=\Phi_{\text {opt }}=0.023, \Psi_{\text {des }}=$ 0.383. This stage is low flow rate and has unusually small loading factor. Close parameters have stages \#\#24. The simplified math model predicts their efficiency pessimistically. In other cases the prediction is more satisfactory. The stages \#\#13, 15 have rather low efficiency. It is connected with too small radial dimensions - constructive imitation. The simplified model cannot react on it.

The second source of information is data on several low and extremely low flow rate intermediate stages of the family XXX, developed still in $20^{\text {th }}$ century.

Up to $\Phi_{\text {des }} \geq 0.01$ the simplified model predicts efficiency satisfactory. The flow rate coefficient $\Phi_{\text {des }}=0.01$ limits the simplified model application.

\section{Conclusion}

Taking into account the fact that the simplified model does not operate with the size and shape of the flow part, but only with design parameters and similarity criteria, the result of verification can be considered positive. It should be noted that the simplified model of efficiency in most cases is less than measured. This is a valuable quality for gas-dynamic calculation, including, for its first stage - variant calculation. A new version of the simplified model of efficiency is included in the programs of the Universal Modeling Method. Our design practice demonstrates its effectiveness.

The calculations were performed using the supercomputer center SCC "Polytechnic". The research was supported by a grant of Russian Science Foundation (project No. 18-7910165).

\section{References}

[1] Y. Galerkin, Turbocompressors. LTD information and publishing center KHT. Moscow. 596 (2010)

[2] Y. Galerkin, K. Soldatova, A. Drozdov, Modern state of the universal modeling for centrifugal compressors. International Conference on Numerical Methods in Industrial Processes. World Academy of science, engineering and technology. Paris -9 1, 242 (2015)

[3] E. Popova, Optimization of the main parameters of turbomachine stages on the basis of mathematical modeling. Dis ... cand. tech. sciences. SPbSPU. - St. Petersburg (1991)

[4] K. Seleznev and Yu. Galerkin 1982 Centrifugal compressors. (Leningrad) 271

[5] A. Rekstin, Y. Popova, A. Ucehovscy Centrifugal compressor stages efficiency analysis by means of the approximate algebraic equations. - Oil and Gas Engineering (OGE-2018). - AIP Conf. Proc. 2007. Omsk. - Russia. -. - 030036-1-030036-11 (2018)

[6] Y. Galerkin, K. Soldatova, Modeling the working process of industrial centrifugal compressors. Scientific fundamentals, stages of development, current state (Publishing house of Polytechnic University) 327 (2011)

[7] Y. Galerkin, K. Soldatova, A. Drozdov, New version of the universal modelling for centrifugal compressor gas dynamic design. Purdue Conference. USA (2014)

[8] Y. Galerkin and K. Soldatova, The application of the Universal Modeling Method to development of centrifugal compressor model stages. Conference "Compressors and their Systems". London. 477-487 (2013)

[9] Y. Galerkin, A. Drozdov, A. Rekstin, K. Soldatova, An alternative method for centrifugal compressor loading factor modelling. Conference "Compressors and their Systems" London (2017)

[10] Y. Galerkin, A. Rekstin, K. Soldatova, A. Drozdov Universal modeling method - the instrument for centrifugal compressor gas dynamic design. ASME Gas Turbine India Conference (2015)

[11] Y. Galerkin, K. Soldatova, Loading factor performance of a centrifugal compressor impeller. Specific features and way of modeling. World Academy of Science, Engineering and Technology. ICMSE 2016: 18th International Conference on Mathematics and Statistical Engineering. - Australia. - Sydney. - 3 12, 822 (2016)

[12] K. Soldatova The application of mathematical models for industrial centrifugal compressor optimal design // ICCMS 2018: 10th International Conference on Computer Modeling and Simulation. - Australia. - Sydney. - Paper ID: 008 (2018)

[13] K. Soldatova Creation of a new mathematical model of the flowing part of centrifugal compressors and a database of model stages. Dis doc. tech. sciences. SPbSPU 357 (2017) 